\title{
Nonconvulsive seizures are common in critically ill children
}

and

N.S. Abend, MD

A.M. Gutierrez-Colina, BA
A.A. Topjian, MD
H. Zhao, MA, MS
R. Guo, MS
M. Donnelly, REEGT
R.R. Clancy, MD
D.J. Dlugos, MD, MSCE

Address correspondence and reprint requests to Dr. Nicholas S. Abend, Division of Neurology, The Children's Hospital of Philadelphia, 34th Street and Civic Center Blvd., Philadelphia, PA 19104

abend@email.chop.edu

\section{ABSTRACT}

Background: Retrospective studies have reported the occurrence of nonconvulsive seizures in critically ill children. We aimed to prospectively determine the incidence and risk factors of nonconvulsive seizures in critically ill children using predetermined EEG monitoring indications and EEG interpretation terminology.

Methods: Critically ill children (non-neonates) with acute encephalopathy underwent continuous EEG monitoring if they met institutional clinical practice criteria. Study enrollment and data collection were prospective. Logistic regression analysis was utilized to identify risk factors for seizure occurrence.

Results: One hundred children were evaluated. Electrographic seizures occurred in 46 and electrographic status epilepticus occurred in 19. Seizures were exclusively nonconvulsive in 32 . The only clinical risk factor for seizure occurrence was younger age $(p=0.03)$. Of patients with seizures, only $52 \%$ had seizures detected in the first hour of monitoring, while $87 \%$ were detected within 24 hours.

Conclusions: Seizures were common in critically ill children with acute encephalopathy. Most were nonconvulsive. Clinical features had little predictive value for seizure occurrence. Further study is needed to confirm these data in independent high-risk populations, to clarify which children are at highest risk for seizures so limited monitoring resources can be allocated optimally, and to determine whether seizure detection and management improves outcome. Neurology ${ }^{\circledR}$ 2011;76:1071-1077

\section{GLOSSARY}

cEEG = continuous EEG monitoring; $\mathbf{C l}=$ confidence interval; $\mathbf{N C S}=$ nonconvulsive seizures; $\mathbf{N C S E}=$ nonconvulsive status epilepticus; $\mathbf{O R}=$ odds ratio; PICU = Pediatric Intensive Care Unit.

Nonconvulsive seizures (NCS) and nonconvulsive status epilepticus (NCSE) denote electroencephalographic seizures without convulsive activity and often manifest as altered mental status or coma. Since there are no clinically evident convulsions, detection of NCS requires, by definition, EEG. Retrospective database studies have reported that NCS and NCSE are common in critically ill adults, and that younger age is a risk factor. ${ }^{1} \mathrm{NCS}$ also occur in critically ill neonates ${ }^{2}$ and infants following surgery for congenital heart defects. ${ }^{3}$ However, epidemiologic features of NCS and NCSE in critically ill non-neonatal children are incompletely understood. Studies of critically ill children in pediatric intensive care units or emergency departments undergoing continuous EEG monitoring (cEEG) have reported NCS or NCSE in 7\%$47 \% .{ }^{4-12}$ Most involved retrospective review of EEG databases, which could potentially lead to ascertainment bias. ${ }^{4-11}$ One prospective study reported that only $7 \%$ of 100 comatose children had seizures detected by cEEG. ${ }^{12}$

In this prospective observational study of critically ill children who underwent clinically indicated EEG monitoring, we aimed to 1) determine the incidence of NCS and NCSE in

\section{Editorial, page 1036}


critically ill children using predefined institutional practice criteria for cEEG, 2) identify clinical features predictive of seizure occurrence, and 3) determine the duration of cEEG required to identify the majority of patients with seizures.

METHODS This was a prospective observational study of infants and children treated in the Pediatric Intensive Care Unit (PICU) of a single tertiary care referral hospital between July 2008 and November 2009 who underwent clinically indicated cEEG. Neonates were excluded. Children were prospectively and consecutively enrolled except for six 1-week blocks when study staff was not available to obtain consent.

Intensive care and neurology physicians received extensive and repeated written and lecture-based education regarding indications for urgent cEEG. Our institution clinical practice criteria for urgent cEEG were persisting altered mental status after a convulsion, altered mental status without a preceding convulsion that either had an unclear etiology or was disproportionate to the known medical condition, and the presence of abnormal movements or vital sign fluctuations. When physicians identified patients meeting these criteria, cEEG was initiated urgently. EEG interpretation was performed by the neurophysiology service and patients were managed by the PICU and Neurology Consult services. Per clinical protocol, patients underwent monitoring for at least 24 hours when screening for NCS, unless they were undergoing therapeutic hypothermia after cardiac arrest, in which case they were monitored for 72 hours, and patients with NCS detected were monitored for 24 hours after their last seizure.

Long-term monitoring was performed using a GrassTelefactor (Grass Technology, West Warwick, RI) video-EEG system. Twenty-one gold-over-silver scalp surface electrodes (Grass Technology) were positioned according to the international 10-20 system and affixed with Collodion adhesive. EEG data were acquired on a portable bedside monitor networked to the hospital's EEG server, allowing review from multiple hospital sites and remotely.

Clinical and EEG data were prospectively collected. Clinical data consisted of underlying general medical diagnoses, prior neurodevelopmental disorders, prior epilepsy, medications, neuroimaging findings, indication for EEG monitoring, EEG findings including seizure occurrence and characteristics, and the impact of cEEG on clinical care. For purposes of the study, after clinical interpretation, to ensure consistency and complete data, the EEG tracings were reinterpreted by one pediatric neurophysiologist using standardized terminology to define seizures, distinguish between NCS and NCSE, and define whether electroencephalographic seizures had any clinical correlate. Electrographic seizures were defined as an abnormal paroxysmal event that was different from the background lasting longer than 10 seconds (or shorter if associated with a clinical change), with a temporal-spatial evolution in morphology, frequency, and amplitude, and with a plausible electrographic field. Electrographic seizures were categorized by location of onset, duration, and coincident clinical manifestations. Periodic epileptiform discharges, defined as repetitive sharp transients, either occurring in isolation or in repetitive runs, but without evolution into discrete electrographic seizures, were not scored as seizures. Consistent with prior definitions, ${ }^{13}$ repetitive nonevolving discharges occurring at less than $3 \mathrm{~Hz}$ were not defined as seizures, and were considered interictal discharges. Time-locked video was available for all studies and was used as needed to aid in artifact identification and to determine whether electrographic seizures had associated clinical correlates. Clinical seizures were defined as abnormal stereotypic and paroxysmal movements associated with an EEG change. Seizures were considered nonconvulsive if there was no clinical change on simultaneous video and no change identified by bedside caregivers. NCSE was defined as a state of impaired consciousness with either a single 30-minute electroencephalographic seizure or a series of recurrent independent electroencephalographic seizures totaling more than 30 minutes in any 1 -hour period ( $50 \%$ seizure burden). If a patient had NCSE at any point during the recording, they were scored as NCSE, even if NCS had occurred initially and then developed into NCSE.

Descriptive statistics are reported as median and interquartile range. Possible risk factors for seizure occurrence were analyzed by logistic regression. A 2 -sided $p$ value $<0.05$ was used to denote statistical significance. Simple logistic regression analyses were performed to model the probability of seizure occurrence using risk factors including age (as a continuous variable), prior epilepsy (yes or no), mental status at cEEG onset (comatose or obtunded/lethargic), prior convulsion, specific etiology (epilepsy or hypoxic-ischemic encephalopathy or traumatic brain injury or stroke or CNS infection or neurosurgery or other structural or other nonstructural), general etiology (epilepsy or acute structural or acute nonstructural), and neuroimaging (normal or diffusely abnormal or multifocal abnormalities or focal abnormality). Coma was defined as the total absence of arousal and evidence of awareness. Comatose patients have no eye opening or nonreflexive movements, either spontaneously or in response to verbal or tactile stimulation, and had absent sleep-wake cycles. Obtunded/lethargic patients had altered mental status but still exhibited some degree of arousal and awareness.

Standard protocol approvals, registrations, and patient consents. Informed written consent to collect data from patients undergoing clinically indicated cEEG was obtained from all patient guardians by the primary investigator who approached families during cEEG. This study was approved by the Children's Hospital of Philadelphia Institutional Review Board.

RESULTS Subject and enrollment characteristics. A total of 101 children met institutional criteria and underwent cEEG in the PICU. One hundred were enrolled. One family declined consent. The median age was 2.9 years (interquartile range $0.8-9.7$ years). Forty-eight patients were male. Indications for cEEG were altered mental status without a prior convulsion $(\mathrm{n}=54)$, altered mental status with a prior convulsion $(n=45)$, abnormal movements $(n=8)$, and vital sign fluctuations $(\mathrm{n}=3)$. All patients with abnormal movements or vital sign fluctuations also had abnormal mental status. Prior convulsions occurred in 4 of the 8 with abnormal movements and none of the 3 with vital sign fluctuations. The primary acute encephalopathy etiologies are listed in the table.

Seizure occurrence. Electrographic seizures occurred during cEEG in 46 of 100 children (46\%, 95\% confidence interval $[\mathrm{CI}]$ for the proportion of children with seizures $0.36-0.56$ ) (figure 1). Of the $46 \mathrm{pa}-$ 


\begin{tabular}{|c|c|c|c|c|}
\hline \multirow{2}{*}{$\begin{array}{l}\text { Table } \\
\text { Variable }\end{array}$} & \multicolumn{4}{|c|}{$\begin{array}{l}\text { Variables associated with NCS and NCSE by univariate } \\
\text { analysis }(n=100)\end{array}$} \\
\hline & & $\begin{array}{l}\text { Seizure present } \\
(\mathrm{n}=46), \mathrm{n}(\%)\end{array}$ & $\begin{array}{l}\text { Seizure absent } \\
(\mathrm{n}=54), \mathrm{n}(\%)\end{array}$ & $\begin{array}{l}\text { Odds ratio } \\
(95 \% \mathrm{Cl})\end{array}$ \\
\hline Age, $y, r$ & n (SD) & $4.23(4.94)$ & $6.96(6.83)$ & $0.93(0.86-0.99)^{a}$ \\
\hline \multicolumn{5}{|c|}{ History of epilepsy } \\
\hline Yes & & $10(22)$ & $10(19)$ & $1.22(0.46-3.26)$ \\
\hline No & & 36 (78) & $44(81)$ & 1 \\
\hline \multicolumn{5}{|c|}{ Etiology of acute encephalopathy } \\
\hline Epilep & & $11(24)$ & $13(24)$ & 1 \\
\hline Hypor & schemic encephalopathy & $12(26)$ & $19(35)$ & $0.75(0.25-2.20)$ \\
\hline CNS $\mathrm{i}$ & ction & $7(15)$ & $3(6)$ & $2.76(0.57-13.29)$ \\
\hline Traun & c brain injury & $2(4)$ & $5(9)$ & $0.47(0.08-2.94)$ \\
\hline Strok & & $5(11)$ & $2(4)$ & $2.96(0.48-18.34)$ \\
\hline Neuro & gery & $2(4)$ & $3(6)$ & $0.79(0.11-5.60)$ \\
\hline Other & cuctural & $3(7)$ & $3(6)$ & $1.18(0.20-7.08)$ \\
\hline Other & nstructural & $4(9)$ & $6(11)$ & 0.79 (0.18-3.53) \\
\hline \multicolumn{5}{|c|}{$\begin{array}{l}\text { Condensed classification of etiology } \\
\text { of acute encephalopathy }\end{array}$} \\
\hline Epilep & & $11(24)$ & $13(24)$ & 1 \\
\hline Acute & uctural ${ }^{b}$ & $31(67)$ & $35(65)$ & $1.05(0.41-2.67)$ \\
\hline Acute & nstructural & $4(9)$ & $6(11)$ & $0.79(0.18-3.53)$ \\
\hline \multicolumn{5}{|c|}{$\begin{array}{l}\text { Convulsive seizure or status } \\
\text { epilepticus prior to cEEG }\end{array}$} \\
\hline Yes & & $29(63)$ & $27(50)$ & $1.71(0.77-3.80)$ \\
\hline No & & $17(37)$ & $27(50)$ & 1 \\
\hline \multicolumn{5}{|c|}{ Mental status at cEEG onset } \\
\hline Letha & /obtunded & $19(41)$ & $25(46)$ & 1 \\
\hline Coma & & $27(59)$ & $29(54)$ & $1.23(0.55-2.71)$ \\
\hline \multicolumn{5}{|c|}{ Neuroimaging } \\
\hline Norm & & $19(41)$ & $25(46)$ & 1 \\
\hline Diffus & bnormality & $12(26)$ & $13(24)$ & $1.22(0.45-3.25)$ \\
\hline Multif & I abnormality & $8(17)$ & $8(15)$ & $1.32(0.42-4.14)$ \\
\hline Focal & ormality & $7(15)$ & $8(15)$ & $1.15(0.36-3.74)$ \\
\hline
\end{tabular}

Abbreviations: $\mathrm{cEEG}$ = continuous EEG monitoring; $\mathrm{Cl}=$ confidence interval; NCS = nonconvulsive seizures; NCSE $=$ nonconvulsive status epilepticus.

a $p<0.05$.

${ }^{b}$ In the condensed classification of etiology of acute encephalopathy, the acute structural category combines hypoxic-ischemic encephalopathy, CNS infection, traumatic brain injury, stroke, neurosurgery, and other structural etiologies.

tients with electrographic seizures, 14 of $46(30 \%)$ had some convulsive seizures in addition to NCS while 32 of 46 (70\%) had only NCS. Among the 46 children with seizures, 27 had NCS but not NCSE while 19 displayed NCSE (figure 1). Of the 27 with NCS but not NCSE, seizures were entirely nonconvulsive in 20. Of the 19 with NCSE, seizures were entirely nonconvulsive in 12 .

Electrographic seizures during cEEG occurred in $52 \%$ (29 of 56) with documented convulsive seizures prior to undergoing cEEG and 39\% (17 of 44) without convulsive seizures prior to cEEG. Seizures constituted status epilepticus in 25\% (14 of 56) with prior convulsive seizures and $11 \%$ (5 of 44) without prior convulsive seizures. Of those without prior convulsions who had seizures, the seizures were entirely nonconvulsive in $82 \%$ (14 of 17) and constituted NCSE in 24\% ( 4 of 17). Prior neuromuscular blockade was administered to $4(6 \%)$ of those with seizures, including 2 of 32 with NCS and 2 of 14 with NCS and electroclinical seizures. Seizures occurred in $56 \%$ (18 of 32) of children less than 1 year old, $43 \%$ (12 of 28 ) of children age $1-5$ years, and $40 \%$ (16 of 40 ) of children older than 5 years.

Risk factors for seizure occurrence. Younger age was the only risk factor for seizure occurrence with odds ratio (OR) of $0.93,95 \%$ CI $0.86-0.99$ ( $p=0.03$ ). The table illustrates mean (SD) for age and the frequency count (percentage) for categorical risk factors in patients with and without seizures, and OR (95\% CI) from logistic regression.

Time to seizure occurrence. The median duration of cEEG for all subjects was 2 days (range 1-71 days). The median duration of cEEG was 3 days (range 1-71 days) in subjects with seizures and 2 days (range 1-4 days) in those without seizures. cEEG duration was 1 day in 34 subjects, 2 days in 28 subjects, 3 days in 16 subjects, and 4 or more days in 22 subjects. Seizures were identified at the onset of cEEG in $4 \%$, in the first hour of cEEG in $52 \%$, and within the first 24 hours in $87 \%$ of children. Duration to seizure identification was similar in patients with convulsive and nonconvulsive seizures and those with only nonconvulsive seizures (figure 2).

DISCUSSION This prospective observational series of critically ill children who fulfilled predetermined criteria to undergo cEEG demonstrated that NCS and NCSE were common, occurring in $46 \%$ of study subjects. Of those with electrographic seizures, $70 \%$ had exclusively NCS, indicating that in the majority of patients, seizures would not have been detected by close clinical observation alone. The only significant risk factor for NCS was younger age, suggesting that if NCS are to be detected, broad screening criteria are required. Seizures are often not detected in the initial hour of EEG, and the majority of children require only 24 hours of cEEG to detect seizures.

The high incidence of NCS and NCSE are consistent with reports based on retrospective database studies $^{5,6,9-11}$ and prospective studies of children with hypoxic-ischemic encephalopathy. ${ }^{7}$ However, our data differ from a recent prospective study that reported only 7 of 100 comatose children who underwent cEEG had electrographic seizures. ${ }^{12}$ Several issues may underlie this difference. First, the subjects 


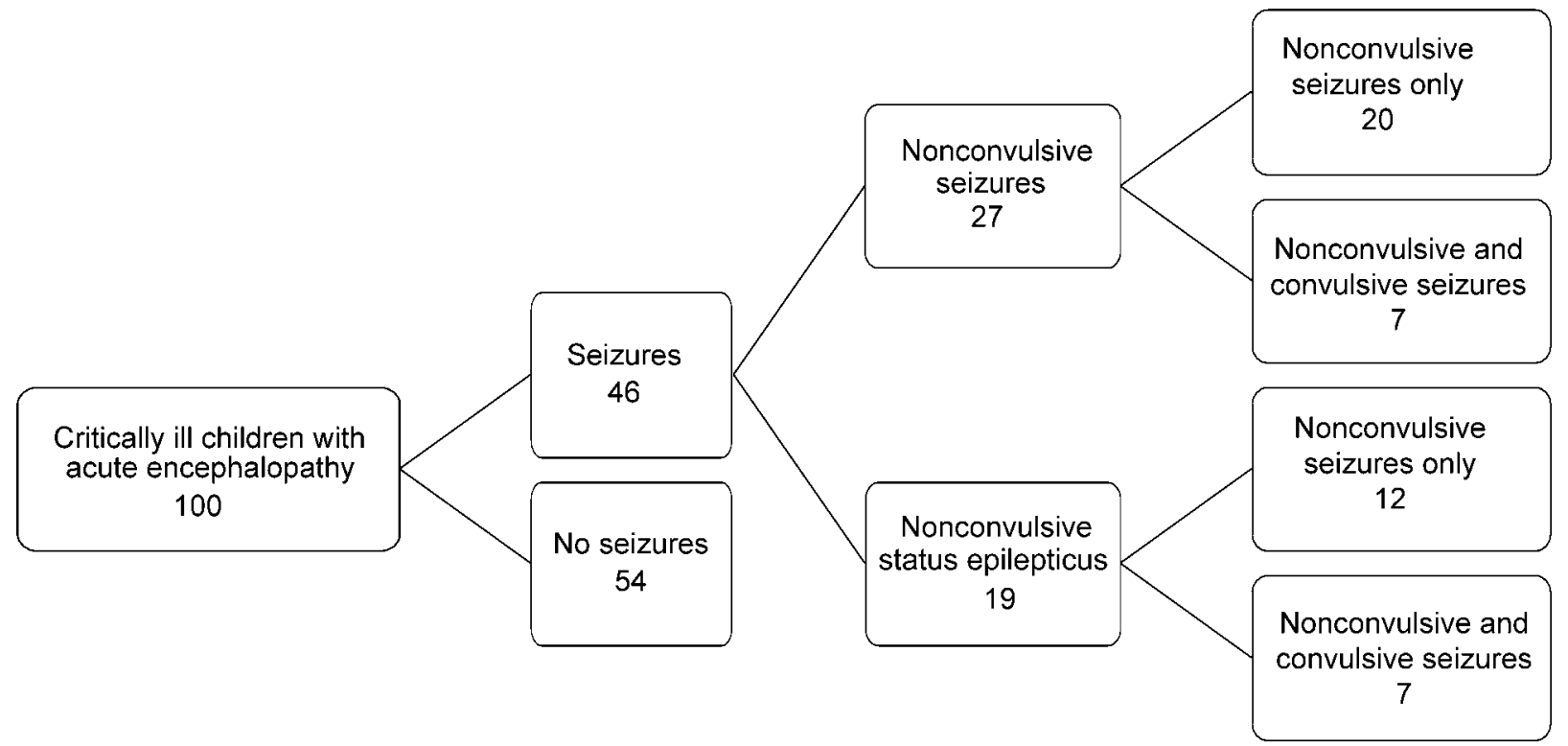

enrolled had different primary medical problems. In the prior study, only half of children had primary brain disorders, and many children undergoing cEEG had respiratory failure, multiorgan dysfunction, or sepsis without specific brain disorders. In our study, 90 of 100 subjects had primary brain disorders and only 10 had nonstructural or non-epilepsyrelated etiologies. Although our clinical protocol for ordering cEEG does not require the presence of a known brain disorder, clinicians may be more likely to order cEEG in that setting. Second, subject screening criteria were different. In the prior study, one researcher screened all PICU admissions to determine whether cEEG was indicated and the indication for cEEG was depressed consciousness (Glasgow Coma Scale score $<8$ ) for all causes, likely leading to broader implementation of cEEG and potentially a lower occurrence of seizures. In the current study, patients undergoing clinically ordered cEEG were eligible for enrollment but not all ICU patients were screened by study staff to determine who should undergo cEEG.

Of the 100 children monitored due to persisting altered mental status, NCS or NCSE were detected in $52 \%$ of children who had clinically evident convulsions prior to being monitored and in 39\% who had no history of clinically evident convulsions. The presence of prior clinically evident seizures was not a risk factor for NCS. Prior studies have also reported the occurrence of NCS and NCSE with or without preceding convulsions or convulsive status epilepticus. ${ }^{6,8,11}$

Most children had seizures identified within 24 hours of cEEG monitoring while only about half of children had seizures detected within 1 hour, showing that many children with NCS or NCSE would not be identified without prolonged cEEG recording. Simi-

Figure 2 Duration between continuous EEG (cEEG) onset and first electrographic seizure

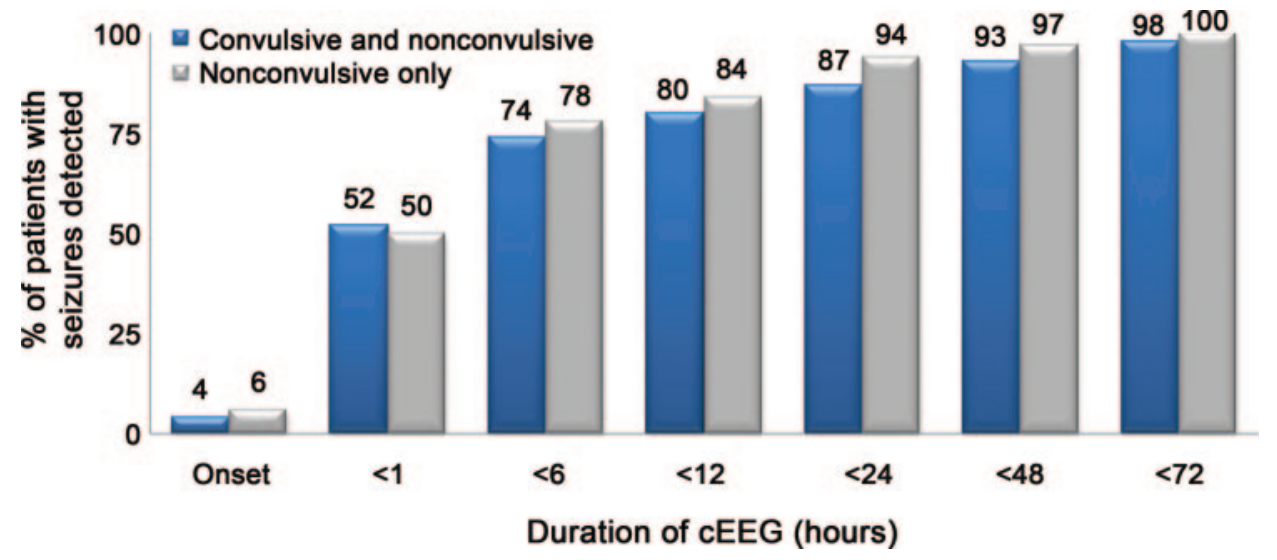


larly, in adults, a 30-minute EEG missed more than half of seizures detected by cEEG, ${ }^{14}$ and in a prospective study of comatose children undergoing cEEG for a median duration of 20.1 hours, all seizures occurred in the first 3 hours. ${ }^{12}$ Infrequent outliers, however, required several days of long-term EEG monitoring, as other studies have also reported. ${ }^{6,8}$ Seizures may occur later in specific clinical situations. For example, a study of children who survived cardiac arrest and underwent therapeutic hypothermia demonstrated that seizure onset often occurred after 24 hours (during the rewarming phase). ${ }^{7}$ Thus, clinical algorithms guiding cEEG utilization may need to account for specific indications and management strategies, and further research is needed to determine the optimal duration of cEEG adjusted for specific clinical or electrographic features.

The only risk factor identified for seizure occurrence was younger age $(p=0.03)$. The $\mathrm{OR}$ was 0.93 , which indicates that for every 1 year increase in age, there was a $7 \%$ decrease in the odds of seizure occurrence. While this was significant, the percent of patients with seizures at each age was not substantially different, suggesting this finding is probably not very helpful clinically in determining which children should undergo cEEG. Other studies have reported that younger age is associated with a higher incidence of NCS and NCSE. Patients less than 18 years of age may be at higher risk than adults for NCSE. ${ }^{1}$ Within the pediatrics age group, neonates and infants may be at particularly high risk. In a study that excluded neonates, $30 \%$ of subjects with NCSE were less than 1 year of age. ${ }^{8}$ In a similar study that included both neonates and children, NCSE was more frequent in those who were less than 1 year old $(36 \%) .{ }^{5}$ In a study of children with altered mental status, 39\% of children with NCS were less than 6 months of age. ${ }^{9}$ Additionally, a study of neonates at risk for seizures found electrographic seizures in 59\%, and of those, $42 \%$ experienced electrographic status epilepticus, many of whom had no clinical seizures. ${ }^{15}$ The underlying etiologies may vary based on age. In one study, none of the patients less than 1 year of age had epilepsy as the etiology of seizures and all had acute structural brain lesions. ${ }^{8}$

Other clinical features considered, including prior epilepsy diagnosis, comatose state vs less severe degree of mental status change, convulsions prior to cEEG, specific and general etiology categories, and imaging findings, were not predictive of seizure occurrence. This suggests that if NCS are to be detected, broad and inclusive cEEG indications will be required. Etiologies resulting in NCS and NCSE are diverse and include hypoxic-ischemic injury, CNS infection, traumatic brain injury, stroke, metabolic disease, and intractable epilepsy with or without re- cent anticonvulsant medication changes. ${ }^{6,8,9,11,12}$ Retrospective studies have reported that $47 \%{ }^{11}$ to $60 \% 8$ of children with NCSE do not have preexisting epilepsy, while a prospective study of comatose children undergoing cEEG reported that 6 of 7 with seizures detected had prior epilepsy diagnoses and witnessed convulsions. ${ }^{12}$ Similar etiologies have been reported in adults. ${ }^{1,16}$

Very few patients with NCS were receiving paralytic drugs. This is similar to studies in neonates that described frequent electrographic-only seizures ${ }^{2}$ and an electro-mechanical uncoupling in which clinical seizures terminated but electrographic seizures persisted after anticonvulsant administration. ${ }^{17}$ This finding demonstrates that many nonparalyzed patients do not show clinical evidence of seizures.

This study has several limitations. First, although physicians received extensive education regarding indications for cEEG monitoring, initiation of cEEG required an order from the PICU or Neurology Consult services, so it is unknown whether every patient who met criteria for cEEG was actually studied. More exact epidemiologic data could be acquired if a prospective patient screening system were utilized in which all PICU patients were evaluated by study personnel every day. The frequency of seizures identified in this prospective study is similar to that identified in prior retrospective studies, ${ }^{5,6,9-11}$ but those too may have been affected by selection bias. As described above, a prospective study of comatose children undergoing cEEG in which a single investigator screened all PICU admissions to determine cEEG eligibility identified a lower seizure occurrence. ${ }^{12}$ Second, while we employed standardized definitions for seizures and status epilepticus, these definitions are variable across studies. Consistent with previous pediatric studies, we defined electrographic seizures as lasting longer than 10 seconds, ${ }^{6,7,9}$ unless there was a clinical correlate associated with briefer electrographic events. However, it is not known whether briefer evolving discharges impact outcome and warrant treatment. ${ }^{18}$ We defined status epilepticus as a single seizure lasting longer than 30 minutes or recurrent seizures totaling more than 30 minutes in any 1-hour period. However, the optimal method for describing recurrent seizures has not been established and it remains unclear whether recurrent seizures have the same impact as a single prolonged seizure. Additionally, variability is reported among neurophysiologists detecting seizures ${ }^{19,20}$ and rhythmic or periodic patterns ${ }^{21}$ in critically ill patients. While EEG tracings in this study were interpreted by a single pediatric neurophysiologist to improve standardization, this may impact generalization of these data. Development of terminology to better define $\mathrm{NCS}^{13}$ 
and periodic and rhythmic discharges ${ }^{22}$ is an important step in eliminating variability in potential future multicenter and multireader studies, and in improving the generalization of study findings. Third, this study included only a few children with each of the risk factors for seizures, likely leading to the wide CIs of most of the risk factor ORs. Development of multicenter databases to track larger numbers of children undergoing cEEG may help identify subpopulations at risk for NCS and NCSE.

The impact of NCS and NCSE on outcome remains unclear. Seizures have been associated with worse outcome in critically ill adults ${ }^{23-25}$ and neonates ${ }^{15,26-29}$ and seizure detection impacts anticonvulsant use in children ${ }^{30}$ and adults. ${ }^{31}$ While plausible mechanisms by which electrographic seizures may lead to brain injury have been described, ${ }^{32,33}$ further study is needed to determine whether identification and treatment of NCS and NCSE improves outcome.

\section{AUTHOR CONTRIBUTIONS}

Statistical analysis was conducted by Huaqing Zhao and Rong Guo.

\section{DISCLOSURE}

Dr. Abend receives research support from the NIH/NINDS. A.M. Gutierrez-Colina reports no disclosures. Dr. Topjian receives research support from the NIH. H. Zhao and R. Guo report no disclosures. M. Donnelly receives research support from the American Society of Electroneurodiagnostics. Dr. Clancy reports no disclosures. Dr. Dlugos receives research support from the NIH/NINDS

Received March 26, 2010. Accepted in final form September 28, 2010.

\section{REFERENCES}

1. Claassen J, Mayer SA, Kowalski RG, Emerson RG, Hirsch LJ. Detection of electrographic seizures with continuous EEG monitoring in critically ill patients. Neurology 2004; 62:1743-1748.

2. Clancy RR, Legido A, Lewis D. Occult neonatal seizures. Epilepsia 1988;29:256-261.

3. Clancy RR, Sharif U, Ichord R, et al. Electrographic neonatal seizures after infant heart surgery. Epilepsia 2005;46: $84-90$.

4. Hyllienmark L, Amark P. Continuous EEG monitoring in a paediatric intensive care unit. Eur J Paediatr Neurol 2007;11:70-75.

5. Hosain SA, Solomon GE, Kobylarz EJ. Electroencephalographic patterns in unresponsive pediatric patients. Pediatr Neurol 2005;32:162-165.

6. Jette N, Claassen J, Emerson RG, Hirsch LJ. Frequency and predictors of nonconvulsive seizures during continuous electroencephalographic monitoring in critically ill children. Arch Neurol 2006;63:1750-1755.

7. Abend NS, Topjian A, Ichord R, et al. Electroencephalographic monitoring during hypothermia after pediatric cardiac arrest. Neurology 2009;72:1931-1940.

8. Abend NS, Dlugos DJ. Nonconvulsive status epilepticus in a pediatric intensive care unit. Pediatr Neurol 2007;37: $165-170$.
9. Saengpattrachai M, Sharma R, Hunjan A, et al. Nonconvulsive seizures in the pediatric intensive care unit: etiology, EEG, and brain imaging findings. Epilepsia 2006;47: $1510-1518$

10. Alehan FK, Morton LD, Pellock JM. Utility of electroencephalography in the pediatric emergency department. J Child Neurol 2001;16:484-487.

11. Tay SK, Hirsch LJ, Leary L, Jette N, Wittman J, Akman CI. Nonconvulsive status epilepticus in children: clinical and EEG characteristics. Epilepsia 2006;47:1504-1509.

12. Shahwan A, Bailey C, Shekerdemian L, Harvey AS. The prevalence of seizures in comatose children in the pediatric intensive care unit: a prospective video-EEG study. Epilepsia 2010;51:1198-1204.

13. Jirsch J, Hirsch LJ. Nonconvulsive seizures: developing a rational approach to the diagnosis and management in the critically ill population. Clin Neurophysiol 2007;118: $1660-1670$.

14. Pandian JD, Cascino GD, So EL, Manno E, Fulgham JR. Digital video-electroencephalographic monitoring in the neurological-neurosurgical intensive care unit: clinical features and outcome. Arch Neurol 2004;61:1090-1094.

15. McBride MC, Laroia N, Guillet R. Electrographic seizures in neonates correlate with poor neurodevelopmental outcome. Neurology 2000;55:506-513.

16. Alroughani R, Javidan M, Qasem A, Alotaibi N. Nonconvulsive status epilepticus; the rate of occurrence in a general hospital. Seizure 2009;18:38-42.

17. Scher MS, Alvin J, Gaus L, Minnigh B, Painter MJ. Uncoupling of EEG-clinical neonatal seizures after antiepileptic drug use. Pediatr Neurol 2003;28:277-280.

18. Chong DJ, Hirsch LJ. Which EEG patterns warrant treatment in the critically ill? Reviewing the evidence for treatment of periodic epileptiform discharges and related patterns. J Clin Neurophysiol 2005;22:79-91.

19. Ronner HE, Ponten SC, Stam CJ, Uitdehaag BM. Interobserver variability of the EEG diagnosis of seizures in comatose patients. Seizure 2009;18:257-263.

20. Abend NS, Gutierrez-Colina AM, Zhao H, et al. Interobserver reproducibility of electroencephalogram interpretation in critically ill children. J Clin Neurophysiol Epub 2011 Jan 10.

21. Gerber PA, Chapman KE, Chung SS, et al. Interobserver agreement in the interpretation of EEG patterns in critically ill adults. J Clin Neurophysiol 2008;25:241-249.

22. Hirsch LJ, Brenner RP, Drislane FW, et al. The ACNS subcommittee on research terminology for continuous EEG monitoring: proposed standardized terminology for rhythmic and periodic EEG patterns encountered in critically ill patients. J Clin Neurophysiol 2005;22:128-135.

23. Young GB, Jordan KG, Doig GS. An assessment of nonconvulsive seizures in the intensive care unit using continuous EEG monitoring: an investigation of variables associated with mortality. Neurology 1996;47:83-89.

24. Oddo M, Carrera E, Claassen J, Mayer SA, Hirsch LJ. Continuous electroencephalography in the medical intensive care unit. Crit Care Med 2009;37:2051-2056.

25. Carrera E, Claassen J, Oddo M, Emerson RG, Mayer SA, Hirsch LJ. Continuous electroencephalographic monitoring in critically ill patients with central nervous system infections. Arch Neurol 2008;65:1612-1618.

26. Glass HC, Glidden D, Jeremy RJ, Barkovich AJ, Ferriero DM, Miller SP. Clinical neonatal seizures are independently associated with outcome in infants at risk for 
hypoxic-ischemic brain injury. J Pediatr 2009;155:318323.

27. Coen RW, McCutchen CB, Wermer D, Snyder J, Gluck FE. Continuous monitoring of the electroencephalogram following perinatal asphyxia. J Pediatr 1982;100:628630.

28. Pisani F, Cerminara C, Fusco C, Sisti L. Neonatal status epilepticus vs recurrent neonatal seizures: clinical findings and outcome. Neurology 2007;69:2177-2185.

29. Ronen GM, Buckley D, Penney S, Streiner DL. Longterm prognosis in children with neonatal seizures: a population-based study. Neurology 2007;69:1816-1822.

30. Abend NS, Topjian AA, Gutierrez-Colina AM, Donnelly M, Clancy RR, Dlugos DJ. Impact of continuous EEG monitoring on clinical management in critically ill children. Neurocrit Care Epub 2010 May 25.

31. Kilbride RD, Costello DJ, Chiappa KH. How seizure detection by continuous electroencephalographic monitoring affects the prescribing of antiepileptic medications. Arch Neurol 2009;66:723-728.

32. Vespa PM, Miller C, McArthur D, et al. Nonconvulsive electrographic seizures after traumatic brain injury result in a delayed, prolonged increase in intracranial pressure and metabolic crisis. Crit Care Med 2007;35:2830-2836.

33. Hauf M, Slotboom J, Nirkko A, von Bredow F, Ozdoba C, Wiest R. Cortical regional hyperperfusion in nonconvulsive status epilepticus measured by dynamic brain perfusion CT. AJNR Am J Neuroradiol 2009;30:693-698.

\section{Visit the Neurology ${ }^{\circledR}$ Web Site at www.neurology.org}

- Enhanced navigation format

- Increased search capability

- Highlighted articles

- Detailed podcast descriptions

- RSS Feeds of current issue and podcasts

- Personal folders for articles and searches

- Mobile device download link

- AAN Web page links

- Links to Neurology Now ${ }^{\circledR}$, Neurology Today ${ }^{\circledR}$, and Continuum ${ }^{\circledR}$

- Resident \& Fellow subsite

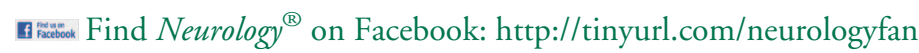

twittor Follow Neurolog $y^{\circledR}$ on Twitter: http://twitter.com/GreenJournal 